\title{
Experimental Organism Benign Polyp
}

National Cancer Institute

\section{Source}

National Cancer Institute. Experimental Organism Benign Polyp. NCI Thesaurus. Code C112275.

A benign polypoid neoplasm of an epithelial lining projecting into a lumen or cavity. (CDISC) 